\title{
Comparação entre abordagens de classificação por pixel e baseada em objeto para o monitoramento de recursos hídricos
}

\author{
Comparison of Pixel Analysis and Object-Based Classification \\ Approaches for Water Resources Monitoring
}

\author{
Emerson Leão Junior ${ }^{1}$ \\ Maria de Lourdes Bueno Trindade Galo ${ }^{1}$ \\ Ivana Ivánová ${ }^{1,2}$
}

Recebido em novembro de 2017.

Aprovado em setembro de 2018.

\begin{abstract}
RESUMO
Considerando o cenário durante a crise hídrica de 2014 e a situação crítica dos reservatórios do sistema Cantareira no estado de São Paulo, este estudo consistiu no monitoramento de mudanças na cobertura da terra na área do reservatório Jaguari-Jacareí a partir de imagens multiespectrais e análise da qualidade temática da informação produzida, utilizando diferentes abordagens de classificação. Na extração dos tipos de cobertura da terra foram utilizadas imagens multiespectrais RapidEye tomadas antes e durante a crise hídrica (2013 e 2014, respectivamente) e avaliadas duas abordagens de classificação supervisionadas: por pixel, baseada no método não paramétrico Support Vector Machine (pixel-SVM) e orientada a objeto por meio de árvore de decisão (OBIA-AD). Na análise da qualidade das classificações foram consideradas as concordâncias e discrepâncias expressas na matriz de confusão de cada classificação obtida, enquanto que a deteç̧ão das mudanças nas margens do reservatório foi realizada por meio da intersecção dos mapas temáticos do corpo hídrico em 2013 e 2014, produzidos individualmente pelos classificadores pixel-SVM e OBIA-AD.No geral, ambos os métodos de classificação produziram informação acurada e adequada para o mapeamento dos tipos de cobertura da terra do entorno do reservatório Jaguari-Jacareí, porém a abordagem baseada em aprendizado de máquina (pixel-SVM) resultou em indicadores de acurácia superiores à classificação OBIA, em 2013 e 2014. A análise das mudanças, que indicou os tipos de cobertura que ocuparam as margens do reservatório com a redução
\end{abstract}

\footnotetext{
${ }^{1}$ Universidade Estadual Paulista Júlio de Mesquita Filho - Unesp, Faculdade de Ciências e Tecnologia - FCT, Programa de Pós-graduação em Ciências Cartográficas, Rua Roberto Simonsen, 305. 19060-900. Presidente Prudente, SP, Brasil. E-mail: emerson.leaojr@gmail.com; mlourdes@fct.unesp.br; i.ivanova@fct.unesp.br

${ }^{2}$ Curtin University, Faculty of Science and Engineering, Department of Spatial Sciences, Kent Street, Bentley, Perth, WA 6102, Australia
} 
da superfície do corpo d'água, mostrou que o classificador pixel-SVM foi mais consistente na atribuição de classes, uma vez que OBIA-AD mapeou parte dessa área como objetos impermeáveis. Em relação à detecção do corpo d'água, ambas as abordagens resultaram em áreas similares em 2013 (35,37 $\mathrm{km}^{2}$ por OBIA-AD contra $36,24 \mathrm{~km}^{2}$ por pixel-SVM) e $2014\left(17,51 \mathrm{~km}^{2}\right.$ por OBIA-AD e $17,46 \mathrm{~km}^{2}$ por pixel-SVM), indicando que teve sua superfície reduzida em quase $50 \%$ com a crise hídrica.

PALAVRAS-CHAVE: Classificação de Imagens. Acurácia de Classificação. Detecção de Mudanças.

\begin{abstract}
This study aims to extract information from multispectral images and to analyse the land cover changes in the area of a water reservoir. The study was motivated by the critical situation of the reservoirs from Cantareira System in São Paulo State of Brazil caused by water crisis in 2014 and our study area was the Jaguari-Jacareí reservoir. This area was extracted as a result of classification of RapidEye multispectral images acquired before and during the water crisis (2013 and 2014, respectively). The information extraction was carried out in two classification approaches: per pixel, based on the non-parametric method Support Vector Machine (pixel-SVM) and object-oriented image analysis through decision tree (OBIA-DT). We evaluated the thematic accuracy of each classification and evaluated the change in the reservoir margins by the intersection of the water body obtained in 2013 and 2014, extracted by the pixel-SVM and OBIA-DT classification. Both classification methods produced accurate land cover maps of the Jaguari-Jacareí reservoir area, but the pixel machine learningbased approach resulted in higher accuracy indicators than the OBIA classification in 2013 and 2014. The change detection analysis, which indicated the coverage types that occupied the reservoir banks with the reduction of the water body surface, showed that the pixel-SVM classifier was more consistent in class assignment, since OBIA-DT mapped part of that area as impervious objects. Regarding water body detection, in both approaches areas calculated for extracted water bodies were similar (35.37 $\mathrm{km} 2$ by OBIA-DT versus $36.24 \mathrm{~km} 2$ by pixel-SVM in 2013$)$ and $(17.51 \mathrm{~km} 2$ by OBIA-DT and $17.46 \mathrm{~km} 2$ by pixel-SVM in 2014), and the results indicate that the surface area of the reservoir was reduced by almost $50 \%$ during the water crisis.
\end{abstract}

KEYWORDS: Image Classification. Classification Accuracy. Change Detection

$$
* * *
$$

\title{
Introdução
}

Nos anos de 2014 e 2015, o sudeste brasileiro enfrentou sua pior seca nos últimos 55 anos (MARENGO et al., 2015), o que culminou com a crise hídrica paulista e comprometimento do abastecimento de água em São Paulo, 
principalmente pelo Sistema Cantareira, que apresentou as maiores evidências deste desastre (NOBRE et al., 2016). A situação em que se encontravam os níveis de água nos reservatórios paulistas em 2014 era tão baixa que foi necessário a utilização de duas cotas da reserva técnica para suprir a demanda da população. Com isso, 210,5 bilhões de litros de água foram retirados do reservatório Jaguari-Jacareí, principal provedor de água do sistema, o que contribuiu para que seu espelho d'água atingisse os menores níveis desde o seu início operacional em 1982 (SABESP, 2015).

O recorrente cenário de baixo nível de água nos reservatórios do Sistema Cantareira aponta para a necessidade de monitorar não apenas a cota d'água dos reservatórios, mas também seu entorno e a superfície do espelho d'água, assim como outras variáveis que possam servir de alerta para uma eventual crise hídrica. Nesse contexto, a disponibilidade de imagens de sensoriamento remoto, algumas delas obtidas sem interrupção desde a década de 1970 por meio de sensores embarcados em plataformas orbitais, constituem uma importante fonte de dados para o monitoramento de recursos sobre a superfície terrestre e desenvolvimento de modelos de predição de catástrofes (JENSEN, 2011).

O mapeamento da superfície terrestre é fundamental para o monitoramento dos recursos naturais e a classificação de imagem é a técnica utilizada para a produção de mapas temáticos a partir de dados de sensoriamento remoto. Com o aumento da capacidade de processamento dos computadores, novos métodos de classificação mais robustos foram desenvolvidos. Dentre as novas abordagens, destacam-se aquelas baseadas em aprendizado de máquina (Machine Learning - ML), como o Support Vector Machine, SVM, (HAN, CHEN e FENG, 2015; KHATAMI, MOUNTRAKIS e STEHMAN, 2016) e a análise de imagem orientada ao objeto, como OBIA, (MYINT et al., 2011; WHITESIDE, BOGGS e MAIER, 2011; DURO, FRANKLIN e DUBÉ, 2012).

Support Vector Machine (SVM) é um classificador supervisionado nãoparamétrico, cuja concepção se baseia na Teoria de Aprendizado estatístico 
eque se utiliza de um hiperplano otimizado no espaço multiespectral para definir a maior distância possível entre duas classes (VAPNIK, 1998). No caso de não haver separação linear das classes, há dois artifícios matemáticos para sua discriminação: inserção de parâmetros de folga, que aumentam a flexibilidade de separação, e/ou utilização de funções de kernel, que mapeia os dados para uma dimensão superior, na qual essas classes possam ser separáveis linearmente (MOUNTRAKIS, IM e OGOLE, 2011).

$\mathrm{Na}$ análise de imagem orientada ao objeto, as classes são atribuídas a grupo de pixels com base em suas características espectrais, espaciais e contextuais. Os objetos são gerados a partir de segmentação da imagem, na qual pixels homogêneos e contíguos são agrupados e passam a representar um mesmo objeto. Desse modo, além da resposta espectral do alvo, outros atributos podem ser utilizados na classificação, como textura, forma e contexto (SCHWIE e TUFTE, 2006). Na classificação dos objetos, diversos métodos podem ser utilizados, inclusive aqueles comumente usados em abordagens por pixel, mas podendo incorporar atributos não-espectrais. Dentre essas possibilidades, destaca-se o método de Árvore de Decisão (AD), o qual se utiliza regras que caracterizam os atributos dos dados para executar a classificação (LALIBERTE, FREDRICKSON e RANGO, 2007).

De acordo com Alsdorf, Rodríguez e Lettenmaier (2007), imagens adquiridas por sensores embarcados em satélites são adequadas ao monitoramento de recursos hídricos, pois permitem extrair informações sobre a variação em área ao longo do tempo. Kalkan et al. (2013), avaliaram o potencial de classificadores baseados em pixels e em objetos na delimitação da superfície de corpos d'água e mostraram que o resultado obtido pela abordagem por pixel se aproximou mais da vetorização manual de imagens. Zhu, Jia e Lv (2014), utilizaram dados provenientes dos satélites ICESat e Landsat-5 para estabelecer relações entre as variações do nível e da área superficial com as variações de volume do lago Qinghai, China, e concluíram que esses dados podem ser utilizados em estimativas que viabilizam o monitoramento de variações no volume de reservatórios. Muala et al. (2014), 
além de utilizar dados de altimetria e imageamento adquiridos por plataformas orbitais para a estimação de volume, também desenvolveram um modelo matemático para inferir o fluxo de água para o lago Nasser e o reservatório Roseires, localizados no Egito e Sudão, respectivamente.

Considerando a possibilidade de se dispor de informação atual e confiável para o monitoramento dos recursos hídricos, adotou-se como estudo de caso a redução drástica no volume de água do reservatório Jaguari-Jacareí, associada à crise hídrica de 2014, e concebeu-se um experimento cujo objetivo é avaliar o desempenho de abordagens de classificação por pixel e baseada em objetos no mapeamento da cobertura da terra e extração da área do espelho d'água, antes e após o evento. Com isso, faz-se uma análise da magnitude da redução do espelho d'água e das transições na cobertura da terra decorrentes do fenômeno.

\section{Materiais e métodos}

A abordagem metodológica desenvolvida considera a premissa de que os produtos obtidos por meio de classificação de imagens orbitais multiespectrais, utilizando-se tanto uma abordagem por pixel quanto orientada a objeto, possuem acurácia adequada para extração de informações espaciais que viabilize a estimativa da redução da superfície aquática e das mudanças nos tipos de cobertura da terra no entorno do reservatório JaguariJacareí, em consequência da crise hídrica de 2014.

A área do entorno do reservatório Jaguari-Jacareí considerada (Figura 1) é delimitada pelas coordenadas geográficas: $22^{\circ} 53^{\prime} 58^{\prime \prime}$ a $23^{\circ} 02^{\prime} 01^{\prime \prime} \mathrm{S}$ e $46^{\circ} 17^{\prime} 56^{\prime \prime}$ a $46^{\circ} 27^{\prime} 27^{\prime} O$. Incluindo, parcialmente, os municípios paulistas de Bragança Paulista, Vargem, Joanópolis e Piracaia. O Jaguari-Jacareí é a maior represa do Sistema Cantareira e principal fornecedora de água à Região Metropolitana de São Paulo. Sua área e volume máximo são, respectivamente, $1230 \mathrm{~km}^{2}$ e $808,12 \mathrm{hm}^{3}$ (ou 808,12 bilhões de litros, já que 1 $\mathrm{hm}^{3}=1$ milhão de $\mathrm{m}^{3}=1$ bilhão de litros). $\mathrm{O}$ início da construção do 
reservatório deu-se no ano de 1977 e sua operação iniciou-se no ano de 1982 (ANA, 2016).

Figura 1 - Localização geográfica do Reservatório Jaguari-Jacareí e delimitação da área de estudo em composição colorida RGB das bandas do espectro visível de imagem

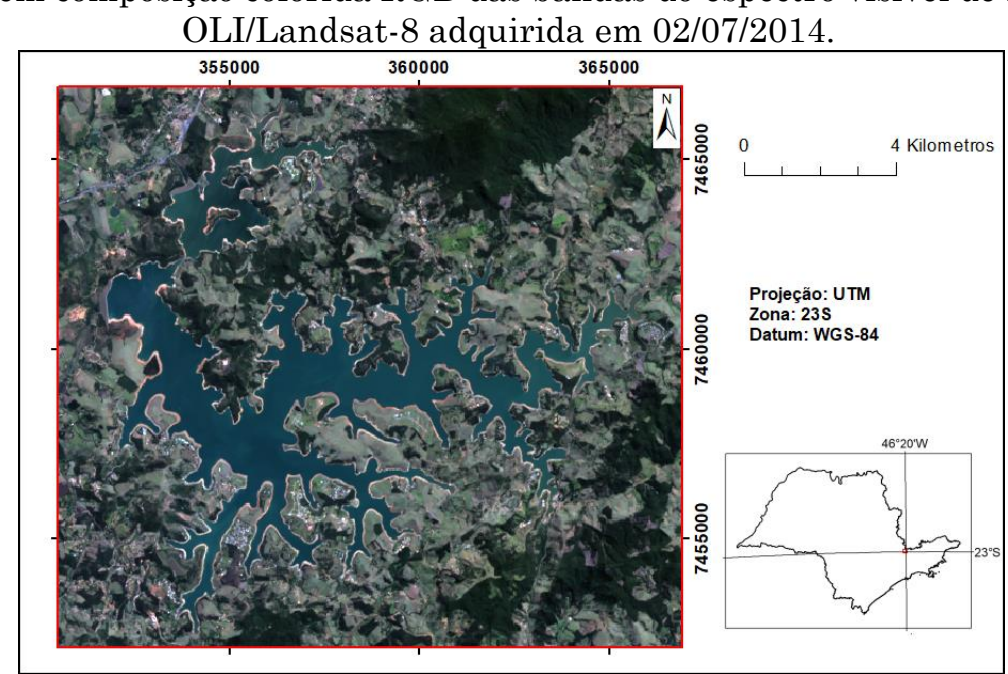

Fonte: Elaborado pelos autores.

Para o estudo, foram utilizadas imagens RapidEye tomadas nos anos de 2013 e 2014, disponibilizadas pelo Ministério do Meio Ambiente (http://geocatalogo.mma.gov.br). Foram necessárias quatro cenas para representar de forma integral o reservatório Jaguari-Jacareí e seu entorno, sendo três delas tomadas no dia 09 de setembro de 2013, uma em 13 de outubro de 2013; três no dia 30 de julho 2014 e uma em 10 de outubro de 2014.

A constelação RapidEye é composta por cinco satélites pertencentes no mesmo plano orbital e seus sensores registram os dados da superfície terrestre em cinco bandas espectrais: azul (440-510 nm), verde (520-590 nm), vermelho (630-685 nm), red edge (690-730 nm) e infravermelho próximo (760$850 \mathrm{~nm}$ ). Os sensores são do tipo pushbroom e adquirem imagens no nadir com tempo de revisita é de 5,5 dias e GSD (Ground Sample Distance) de 6,5 metros na aquisição no nadir (PLANET, 2016). O Ministério do Meio Ambiente disponibiliza imagens ortorretificadas que incorporam correções geométricas, dos efeitos de inclinação da plataforma de aquisição e utilizam, além disso, pontos de controle de terreno e modelos digitais de superfície 
(MDS) precisos para a geração de ortofotos, na projeção UTM no sistema de referência WGS 84. Com isso, o tamanho do pixel da imagem ortorretificada é de 5 metros e sua acurácia espacial global de 10 metros.

Antes de executar a etapa de mapeamento, foi necessário especificar e caracterizar os tipos de cobertura da terra presentes a área de estudos. Essa definição foi feita com base em consulta aos planos diretores dos municípios de Bragança Paulista, Piracaia, Vargem e Joanópolis (PREFEITURA MUNICIPAL DE BRAGANÇA PAULISTA, 2007; PREFEITURA MUNICIPAL DE PIRACAIA, 2007; PREFEITURA MUNICIPAL DE VARGEM, 2000; CÂMARA MUNICIPAL DA INSTÂNICA TURÍSITICA DE JOANÓPOLIS, 2006), imagens históricas disponíveis no GoogleEarth ${ }^{\mathrm{TM}}$ e a interpretação das próprias imagens RapidEye. As classes de cobertura da terra utilizadas foram: Água $(A g)$, constituída pelo Reservatório JaguariJacareí e pequenos corpos d'água; Vegetação Arbórea (VA), áreas cobertas por vegetação de maior porte; Vegetação Rasteira $(V R)$, áreas de vegetação de pequeno porte; Solo Exposto (SE), áreas sem vegetação; e Área Impermeável (Im), que incluiu telhados, infraestrutura do reservatório, pavimentação e rochas expostas.

O processamento das imagens incluiu o registro entre as imagens das duas datas, as classificações individuais das cenas de cada data, usando ambas as abordagens propostas, e o mosaico das imagens classificadas. Optou-se por realizar o mosaico das imagens já classificadas pelo fato de que, mesmo com a normalização radiométrica das imagens que compõem a área de estudos em cada ano, não foi possível homogeneizar as variações radiométricas intrínsecas de alguns alvos (como corpo d'água). Assim, cada uma das quatro imagens RapidEye que compõem a área de estudo foi classificada individualmente, utilizando ambas as abordagens de análise (pixel-SVM e OBIA-AD), para as tomadas de imagens de 2013 e 2014.

Como o SVM é um método de classificação supervisionado foi necessária a aquisição de um conjunto de treinamento que fosse representativo de cada um dos cinco tipos de cobertura da terra. A 
classificação foi feita no software ENVI 5.1, sendo que as amostras de treinamento foram adquiridas em cada imagem por meio da ferramenta 'regiões de interesse' (ROI - regions of interest).

Devido à dificuldade em definir a priori os parâmetros exigidos pelo algoritmo que fornecerão a melhor discriminação dos dados no espaço multiespectral, foram testadas todas as funções de kernel disponíveis no ENVI 5.1, bem como os parâmetros associados a cada uma. Os valores testados para o parâmetro de penalidade $C$ foram: 1, 10, 100 e 200, e para o coeficiente $\gamma$ foram utilizados: 0,$1 ; 0,2 ; 0,5$ e 0,9. Como forma de avaliar cada configuração do SVM, foram utilizadas amostras adicionais para validar as classificações, adotando-se como critério para selecionar o resultado, o maior valor do índice de concordância Kappa. A configuração que melhor se adaptou ao conjunto de dados foi a função kernel de função de base radial, com coeficiente $\gamma$ e parâmetro de penalidade $C$, respectivamente, iguais a $0,1 \mathrm{e}$ 100.

A análise de imagem orientada ao objeto foi desenvolvida no software eCognition Developer 9.0e partiu da delimitação prévia dos objetos na imagem por meio de uma segmentação multirresolução. Para tanto, foram avaliadas diversas configurações de parâmetros, com a finalidade de especificar aqueles que melhor se adequaram ao esquema de hierárquico concebido (Figura 2), no qual, inicialmente, objetos relacionados às superclasses água e não-água foram identificados. No nível de classificação seguinte, foi feita a distinção entre áreas vegetadas e não vegetadas, finalizando com a discriminação entre os tipos de vegetação rasteira e arbórea, bem como entre áreas impermeáveis e solo exposto. Com base nas delimitações dos objetos de interesse obtidas em testes realizados com diferentes configurações de parâmetros, a segmentação foi realizada em apenas um nível de discriminação, atribuiu-se o valor 100 para o parâmetro de escala, 0,5 para compacidade e 0,5 para forma.

A atribuição de classes aos objetos foi realizada por Árvore de Decisão (AD), na qual foram definidas e aplicadas regras que consideraram tanto 
atributos espectrais, como de forma e/ou de localização, como pode ser visto na Figura 2. Devido a diferenças de iluminação entre as cenas, o limiar referente a cada classe é variável por imagem, mas as regras de pertinência se mantiveram para todas as classes, apenas os parâmetros usados variaram de acordo com as características da cena.

Figura 2 - Hierarquia das classes e regras de decisão adotadas na classificação Árvore de Decisão. Ag se refere a classe Corpo d’Água; VA, Vegetação Arbórea; VR, Vegetação Rasteira; SE, Solo Exposto e Im, Área Impermeável

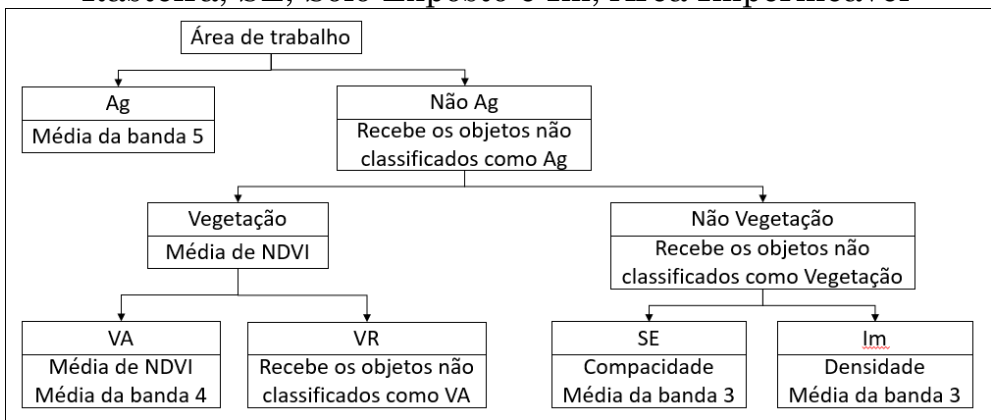

Fonte: Elaborado pelos autores.

A acurácia temática foi estimada por meio de informações derivadas da matriz de confusão, relacionando cada uma das classificações com uma referência, adotada como verdade terrestre, e construída a partir da identificação dos tipos de cobertura da terra em pontos amostrais previamente definidos. O tamanho da amostra, usado para verificação da concordância/discordância entre os elementos amostrais de referência e respectivos elementos extraídos das classificações do mesmo ano, considerou uma precisão desejada (B) de 95\% e nível de significância (a) igual a 5\%, e ficou definindo em 662 pontos amostrais, distribuídos de forma estratificada e aleatória na área de estudos. A referência quanto ao verdadeiro tipo de cobertura da terra foi especificada por meio da interpretação de imagens histórias do GoogleEarth ${ }^{\mathrm{TM}}$, selecionando-se aquelas que mais se aproximavam das datas de tomada dos dados em 2013 e 2014.

A partir da matriz de confusão foram estimadas a acurácia global e por classe (acurácia do usuário e do produtor), além do índice de concordância Kappa (K) para cada ano e método de classificação adotado. $\mathrm{O}$ valor de $\mathrm{K}$ é 
calculado assumindo que a disposição espacial dos elementos amostrais obedece a distribuição normal e que o tamanho da amostra seja grande (CONGALTON e GREEN, 1999). Portanto, pode ser utilizado avaliar a significância de uma matriz de confusão e verificar se a concordância entre a classificação e a referência de campo é significativamente maior do que 0 , indicando se o resultado obtido é melhor do que a atribuição de forma aleatória de classes aos dados de sensoriamento remoto. $\mathrm{O}$ teste de significância da matriz de confusão é expresso pela Equação 1.

$$
\mathrm{Z}=\frac{\mathrm{K}}{\sqrt{\operatorname{var}(\mathrm{K})}}
$$

sendo que $Z$ é um valor padronizado e tem distribuição normal. Nesse teste, assume-se $H_{0}: K=0$ como sendo a hipótese nula e a $H_{1}: K \neq 0$ como alternativa, $H_{o}$ é rejeitada se $Z \geq Z_{\alpha / 2}$, para a/2 nível de significância do teste bicaudal.

Para verificar se as classificações possuíam desempenho similar, foi utilizada a Equação 2, a qual permite comparar as matrizes de confusão resultantes de duas classificações.

$$
\mathrm{Z}=\frac{\left|\mathrm{K}_{1}-\mathrm{K}_{2}\right|}{\sqrt{\operatorname{var}\left(\mathrm{K}_{1}\right)+\operatorname{var}\left(\mathrm{K}_{2}\right)}}
$$

sendo que $Z$ é padronizado e normalmente distribuído. Dado que a hipótese nula $H_{0}:\left(K_{1}-K_{2}\right)=0$, e a alternativa $H_{0}:\left(K_{1}-K_{2}\right) \neq 0, H_{0}$ é rejeitada se $Z \geq$ $Z_{a / 2}$. O nível de confiança adotado neste estudo foi de $95 \%$.

\section{Resultados e discussão}

Os resultados das classificações das imagens RapidEye são apresentados na Figura 3, na qual estão indicados os mapas temáticos obtidos a partir da classificação pixel-SVM aplicada às imagens tomadas em 2013 (a) e 2014 (b), e OBIA-AD das cenas de 2013 (c) e 2014 (d). A denominação das 
classes expressas na legenda dessa figura representa: Ag - Corpo d'Água, VA - Vegetação Arbórea, VR - Vegetação Rasteira, SE - Solo Exposto e Im - Área Impermeável.

Figura 3 - Mapas de cobertura da terra do entorno do reservatório Jaguari-Jacareí elaborados a partir da classificação das imagens RapidEye pela abordagem pixel-SVM, de 2013 (a) e 2014 (b); e OBI-AD, 2013 (c) e 2014 (d).

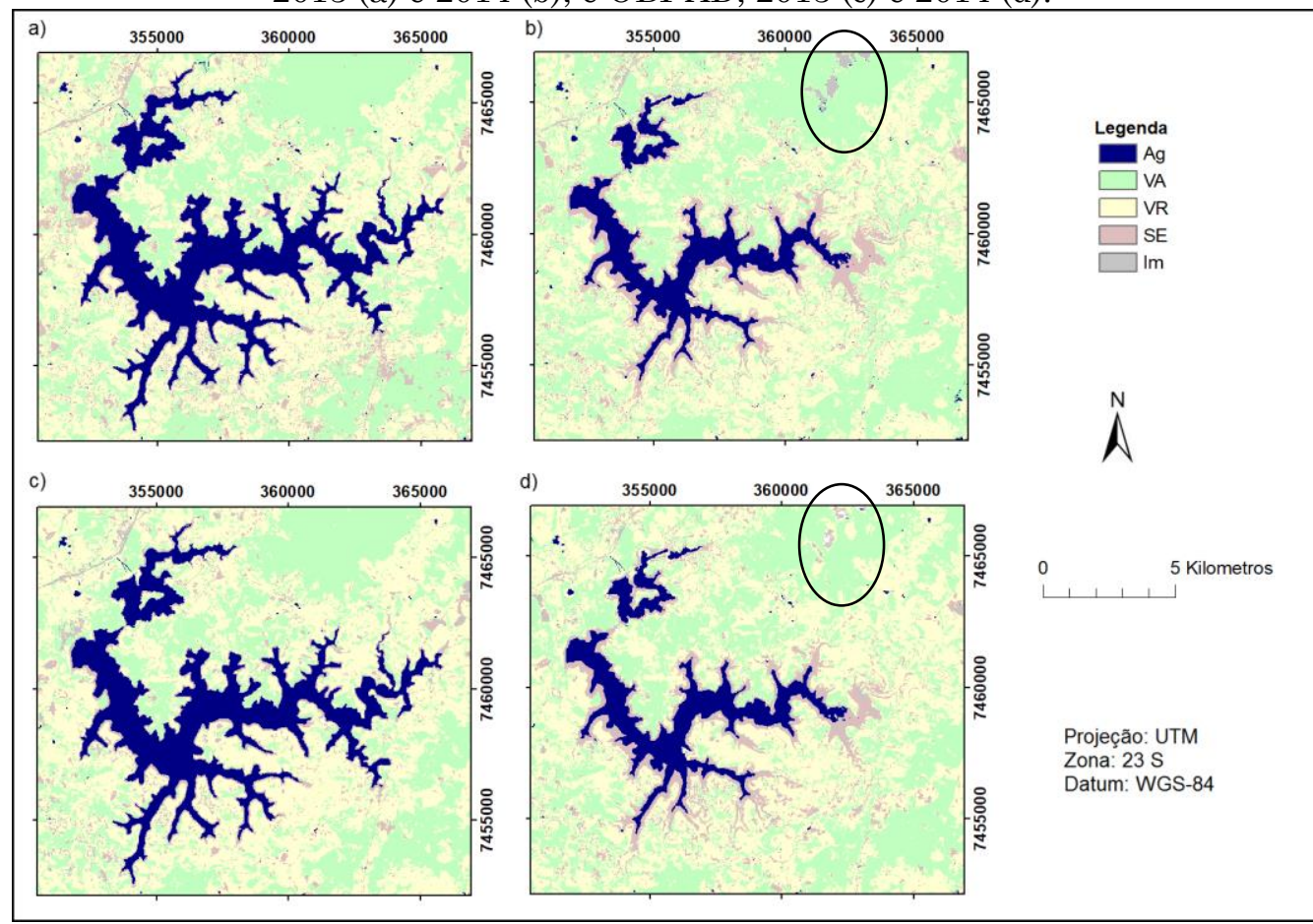

Fonte: Elaborado pelos autores.

O cenário apresentado na

Figura 3 ilustra a expressiva redução na superfície do espelho d’água do reservatório Jaguari-Jacareí de 2013 para 2014, independente da abordagem de classificação adotada. Diferenças na definição espacial dos tipos de cobertura da terra associadas aos métodos de classificação são menos significativas, mas percebe-se que na região demarcada por uma elipse nas classificações de 2014 (pixel-SVM, Figura 3b e OBIA-AD, Figura 3d) uma área com cobertura de nuvens foi atribuída a classes distintas pelos dois classificadores. 
A fim de validar o resultado das classificações, por meio da acurácia temática das informações extraídas, foi construída uma matriz de confusão para cada classificação, indicando o grau de correspondência de cada uma com os dados de referência extraídos de imagens históricas do GoogleEarth ${ }^{\mathrm{TM}}$ de 2013 e 2014, a partir de uma amostra constituída 662 elementos. As matrizes de confusão são apresentadas na Tabela 1, referentes às classificações pixelSVM e OBIA-AD aplicadas às imagens de 2013 e 2014.

Tabela 1 - Matrizes de confusão e índices de concordância kappa das classificações PIXELSVM e OBIA-AD, obtidas para os dados de 2013 e 2014.

\begin{tabular}{|c|c|c|c|c|c|c|c|c|c|c|c|c|c|c|}
\hline \multicolumn{15}{|c|}{ REFERÊNCIA } \\
\hline \multirow{9}{*}{ 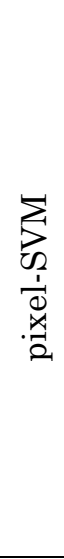 } & \multicolumn{7}{|c|}{2013} & \multicolumn{7}{|c|}{2014} \\
\hline & & $\begin{array}{l}\mathbf{A} \\
\mathrm{g}\end{array}$ & VA & VR & $\begin{array}{l}\mathbf{S} \\
\mathbf{E}\end{array}$ & $\begin{array}{c}\mathbf{I} \\
\mathbf{m} \\
\end{array}$ & $\begin{array}{c}\text { Tota } \\
1\end{array}$ & & $\begin{array}{l}\mathbf{A} \\
\mathbf{g}\end{array}$ & VA & VR & $\begin{array}{l}\mathbf{S} \\
\mathbf{E}\end{array}$ & $\begin{array}{c}\mathbf{I} \\
\mathbf{m}\end{array}$ & $\begin{array}{c}\text { Tota } \\
1\end{array}$ \\
\hline & $\mathrm{Ag}$ & 90 & 0 & 0 & 0 & 0 & 90 & $\mathrm{Ag}$ & 50 & 0 & 0 & 1 & 0 & 51 \\
\hline & VA & 0 & $\begin{array}{c}20 \\
7\end{array}$ & 6 & 0 & 0 & 213 & VA & 0 & $\begin{array}{c}23 \\
8\end{array}$ & 17 & 1 & 0 & 256 \\
\hline & VR & 0 & 6 & $\begin{array}{c}20 \\
7\end{array}$ & 11 & 3 & 227 & VR & 0 & 2 & $\begin{array}{c}22 \\
0\end{array}$ & 5 & 2 & 229 \\
\hline & $\mathrm{SE}$ & 1 & 2 & 25 & 57 & 24 & 107 & $\mathrm{SE}$ & 0 & 0 & 6 & 81 & 13 & 100 \\
\hline & $\mathrm{Im}$ & 0 & 0 & 0 & 10 & 13 & 23 & $\mathrm{Im}$ & 0 & 0 & 2 & 6 & 18 & 26 \\
\hline & $\begin{array}{c}\text { Tota } \\
1\end{array}$ & 91 & $\begin{array}{c}21 \\
5 \\
\end{array}$ & $\begin{array}{c}23 \\
8 \\
\end{array}$ & 78 & 40 & 662 & $\begin{array}{c}\text { Tota } \\
1\end{array}$ & 50 & $\begin{array}{c}24 \\
0\end{array}$ & $\begin{array}{c}24 \\
5\end{array}$ & 94 & 33 & 662 \\
\hline & \multicolumn{7}{|c|}{ Indice Kappa: 0,818 } & \multicolumn{7}{|c|}{ Indice Kappa: 0,882 } \\
\hline \multirow{9}{*}{ 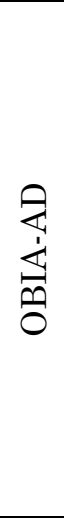 } & \multicolumn{7}{|c|}{2013} & \multicolumn{7}{|c|}{2014} \\
\hline & & $\mathrm{Ag}$ & VA & VR & $\mathrm{SE}$ & $\mathrm{Im}$ & Total & & $\mathrm{Ag}$ & $\mathrm{VA}$ & VR & $\mathrm{SE}$ & $\mathrm{Im}$ & Total \\
\hline & $\mathrm{Ag}$ & 90 & 0 & 0 & 0 & 0 & 90 & $\mathrm{Ag}$ & 49 & 0 & 0 & 1 & 0 & 50 \\
\hline & VA & 0 & $\begin{array}{c}19 \\
3 \\
\end{array}$ & 7 & 0 & 1 & 201 & VA & 0 & $\begin{array}{c}23 \\
0 \\
\end{array}$ & 18 & 0 & 0 & 248 \\
\hline & VR & 0 & 22 & $\begin{array}{c}21 \\
9\end{array}$ & 20 & 14 & 275 & VR & 0 & 10 & $\begin{array}{c}21 \\
6\end{array}$ & 11 & 10 & 247 \\
\hline & $\mathrm{SE}$ & 0 & 0 & 8 & 47 & 9 & 64 & $\mathrm{SE}$ & 1 & 0 & 6 & 67 & 6 & 80 \\
\hline & $\mathrm{Im}$ & 1 & 0 & 4 & 11 & 16 & 32 & $\mathrm{Im}$ & 0 & 0 & 5 & 15 & 17 & 23 \\
\hline & $\begin{array}{c}\text { Tota } \\
1\end{array}$ & 91 & $\begin{array}{c}21 \\
5\end{array}$ & $\begin{array}{c}23 \\
8\end{array}$ & 78 & 40 & 662 & $\begin{array}{c}\text { Tota } \\
1\end{array}$ & 50 & $\begin{array}{c}24 \\
0\end{array}$ & $\begin{array}{c}24 \\
5\end{array}$ & 94 & 33 & 662 \\
\hline & \multicolumn{7}{|c|}{ Indice Kappa: 0,796 } & \multicolumn{7}{|c|}{ Indice Kappa: 0,819 } \\
\hline
\end{tabular}

Fonte: Elaborado pelos autores.

Pela Tabela 1 verifica-se que a classe Área Impermeável (Im) foi aquela que mais se confundiu com as demais, principalmente com Solo Exposto $(S E)$, em ambas as abordagens de classificação e, em menor grau, com Vegetação Rasteira (VR), no método OBIA-AD. De acordo com Herold e Roberts (2010), telhados cerâmicos de material avermelhado apresentam resposta espectral 
semelhante ao solo exposto, razão da confusão observada entre esses tipos de cobertura. Também em menor grau foi observada confusão entre as classes SE (Solo Exposto) e $V R$ (Vegetação Rasteira); e $V A$ (Vegetação Arbórea) com $V R$. A classe $A g$ (Corpo d'Água) apresenta pouca confusão com as demais, devido a resposta espectral sutis que apresenta. Portanto, a extração de corpos d'água a partir de classificação de imagens multiespectrais nas bandas do infravermelho é realizada, praticamente, na sua íntegra.

Em termos de indicadores globais da acurácia, representado pelo Índice Kappa, a classificação pixel-SVM resultou em valores ligeiramente superiores $(0,818$ e 0,882 , respectivamente para 2013 e 2014) que a abordagem OBIA$\mathrm{AD}(0,796$ para 2014 e 0,819 para 2014). Ambos os métodos resultaram em melhor acurácia para a imagem de 2014. Comportamento similar pode ser observado à acurácia global, expressa na Tabela 2, juntamente com as acurácias do usuário e do produtor, por classe.

Tabela 2 - Acurácias do usuário e do produtor por classe resultante das abordagens pixelSVM e OBIA-AD, para cada ano de aquisição da imagem (2013 e 2014)

\begin{tabular}{|c|c|c|c|c|c|c|c|c|c|c|c|c|}
\hline \multicolumn{13}{|c|}{2013} \\
\hline \multicolumn{6}{|c|}{ Acurácia do usuário (\%) } & \multicolumn{6}{|c|}{ Acurácia do Produtor (\%) } & \multirow[b]{2}{*}{ Global } \\
\hline & $\mathrm{Ag}$ & VA & VR & SE & Im & & $\mathrm{Ag}$ & VA & VR & SE & Im & \\
\hline pixel-SVM & 99 & 96 & 87 & 73 & 32 & pixel-SVM & 100 & 97 & 91 & 52 & 56 & 86,7 \\
\hline OBIA-AD & 99 & 90 & 92 & 60 & 40 & OBIA-AD & 100 & 96 & 80 & 73 & 50 & 85,3 \\
\hline \multicolumn{13}{|c|}{2014} \\
\hline \multicolumn{6}{|c|}{ Acurácia do usuário (\%) } & \multicolumn{6}{|c|}{ Acurácia do Produtor (\%) } & \multirow[b]{2}{*}{ Global } \\
\hline & Ag & VA & VR & SE & Im & & Ag & VA & VR & SE & Im & \\
\hline pixel-SVM & 100 & 99 & 90 & 86 & 55 & pixel-SVM & 98 & 93 & 96 & 81 & 69 & 91,7 \\
\hline OBIA-AD & 98 & 96 & 88 & 71 & 51 & OBIA-AD & 98 & 93 & 87 & 84 & 46 & 87,5 \\
\hline
\end{tabular}

As Tabelas 1 e 2 mostram que, para todas as classificações, as classes Im (Área Impermeável) e $S E$ (Solo Exposto) apresentaram menor acurácia, seja do usuário ou do produtor. As demais classes tiveram acurácia do usuário e do produtor superior a 75\%. Os resultados da análise da acurácia indicam uma leve superioridade, em termos de desempenho, da classificação pixelSVM em relação à OBIA-AD, neste estudo especificamente, tanto para os indicadores globais como para as acurácias do usuário e do produtor, por 
classe. O desempenho ligeiramente inferior das classificações OBIA-AD pode ser atribuído, entre outras causas, à própria definição dos objetos, uma vez que a segmentação da imagem utiliza um conjunto de parâmetros de difícil especificação, tendo sido necessária a realização de vários testes para sua definição. Por outro lado, o algoritmo SVM tem mostrado alta eficiência em tarefas de reconhecimento de padrões e, como essas classificações foram baseadas na análise de pixels, uma adequada aquisição de dados de treinamento influenciou positivamente no resultado obtido com essa abordagem.

Foram realizados testes estatísticos para avaliar a significância das diferenças entre as classificações, a partir dos valores de índice $\operatorname{Kappa(K)}$ e variância de Kappa $(\operatorname{var}(\mathrm{K}))$, estimados por meio da Equação 1. Além disso, utilizou-se a Equação 2 para verificar se pares de classificações tiveram desempenho similar. Os resultados obtidos são mostrados na Tabela 3.

Tabela 3 - Variância de Kappa (var (K)), valores de z e do teste de significância, usados para

\begin{tabular}{c|c|c|c|c}
\multicolumn{1}{c}{} & \multicolumn{5}{c}{ avaliar a similaridade entre pares de classificações. } \\
\cline { 2 - 5 } & OBIA-AD $_{\mathbf{2 0 1 3}}$ & pixel-SVM $_{\mathbf{2 0 1 3}}$ & OBIA-AD $_{\mathbf{2 1 4}}$ & pixel-SVM $_{\mathbf{2 0 1 4}}$ \\
\hline $\operatorname{var}(\mathrm{K})$ & 0,00035 & 0,00031 & 0,00033 & 0,00023 \\
\hline OBIA-AD $_{2013}$ & 42,367 & - & - & - \\
\hline pixel-SVM $_{2013}$ & 0,86 & 46,610 & - & - \\
\hline OBIA-AD $_{2014}$ & 0,96 & 0,12 & 45,471 & - \\
\hline pixel-SVM & & & \\
\hline
\end{tabular}

Fonte: Elaborado pelos autores.

As classificações foram analisadas a um nível de confiança de 95\% (Ztabelado igual a 1,96) e como os valores de $\mathrm{Z}$ calculado (realçados na diagonal da Tabela 3) foram superiores ao tabelado em todos os casos, todas as classificações foram significativas. A análise da similaridade entre pares de classificação mostra que, para o mesmo nível de confiança, as classificações podem ser consideradas estatisticamente similares, com exceção do pixelSVM aplicado aos dados de 2014, que teve um desempenho estatisticamente superior às demais e menor variância.

Os efeitos da seca na redução da superfície de água são perceptíveis na 
Figura 3. A área do reservatório obtida pela classificação OBIA-AD dos dados de 2013 foi de $35,37 \mathrm{~km}^{2}$ e de $17,52 \mathrm{~km}^{2}$ para os dados de 2014, resultando em uma diferença de área de $17,85 \mathrm{~km}^{2}$. Em relação à abordagem pixel-SVM, a área do corpo d'água foi de 36,24 e $17,46 \mathrm{~km}^{2}$, respectivamente para 2013 e 2014 (diferença de 18,46). Em ambas as abordagens de classificação, aproximadamente a metade da área do espelho d'água do reservatório em 2013 se manteve em 2014.

Para mostrar os tipos de cobertura da terra que, em 2014 substituíram a classe corpo d água nas margens do reservatório Jaguari-Jacareí, foi realizada uma operação cruzada entre as classificações de 2013 e 2014, resultando nos mapas de transição espaço-temporal indicados na Figura 4 para cada método de classificação: pixel-SVM (a) e OBIA-AD (b). Em ambas as classificações, as classes de transição foram denominadas: $A g$ (corpo d'água que se manteve inalterado), $A g V A$ (transição de água para vegetação arbórea), $A g V R$ (transição de água para vegetação rasteira), AgSE (transição de água para solo exposto) e AgIm (transição de água para área impermeável).

Figura 4 - Tipos de cobertura da terra presentes na área inundada do reservatório JaguariJacareí com a redução do corpo d’água em 2014, definidos pelas abordagens de classificação pixel-SVM (a) e OBIA-AD (b).

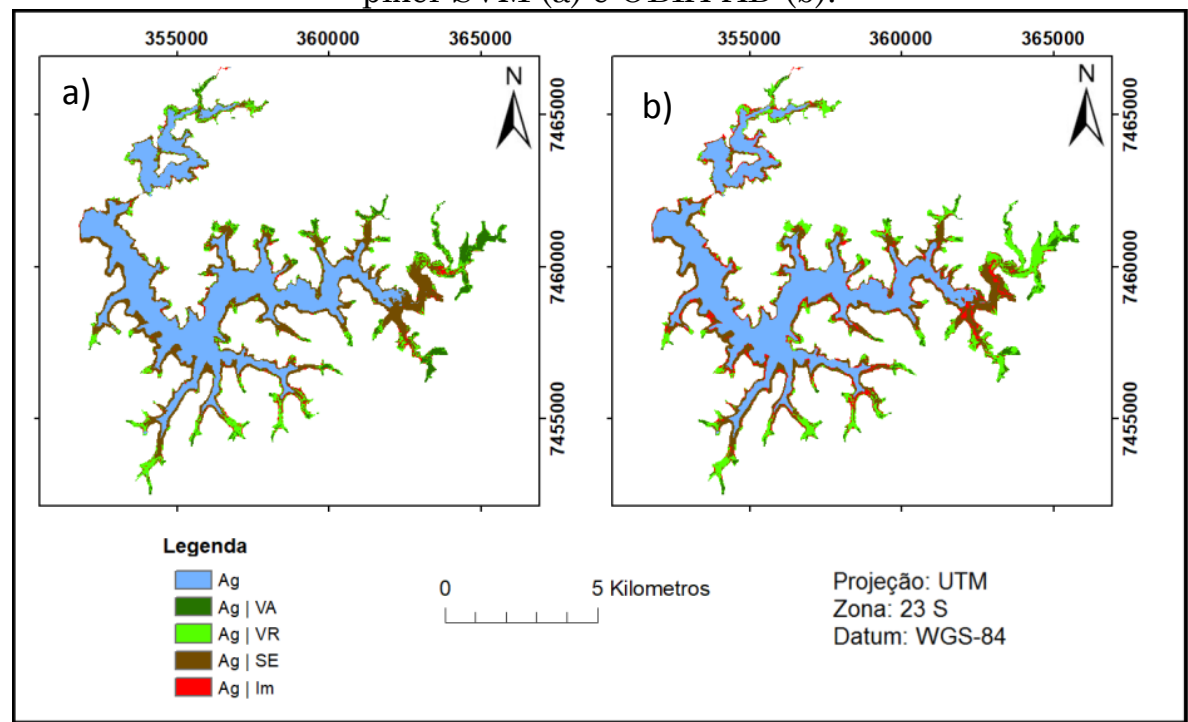

Fonte: Elaborado pelos autores. 
A Figura 4-b (OBIA-AD) indica uma área considerável como transição entre as classes água e área impermeável (AgIm), inconsistente com a realidade, pois as coberturas impermeáveis na cena estão restritas às instalações da represa, píeres e ao afloramento rochoso do canal que interliga os rios Jaguari e Jacareí. Por outro lado, no mapa de transições resultante da classificação pixel-SVM (Figura 4-a), ocorreu uma área considerável associada à transição do corpo d'água com Vegetação Arbórea ( $A g V a)$. De fato, em alguns locais nas margens do reservatório, observa-se a alternância de água para arbustos e a atribuição dessas áreas a vegetação de maior porte textura da vegetação arbustiva, mais próxima de vegetação arbórea do que da rasteira. A fim de quantificar essas transições, foi elaborado o gráfico de barras, Figura 5, mostrando a proporção de mudança da classe Corpo d'Água, mapeado em 2013, para as demais classes (mapeadas em 2014).

Figura 5 - Proporção de mudança da classe corpo d'água para vegetação arbórea (AgVA); vegetação rasteira (AgVR); solo exposto (AgSE) e área impermeável (AgIm), definidos pelas abordagens de classificação pixel-SVM e OBIA-AD.

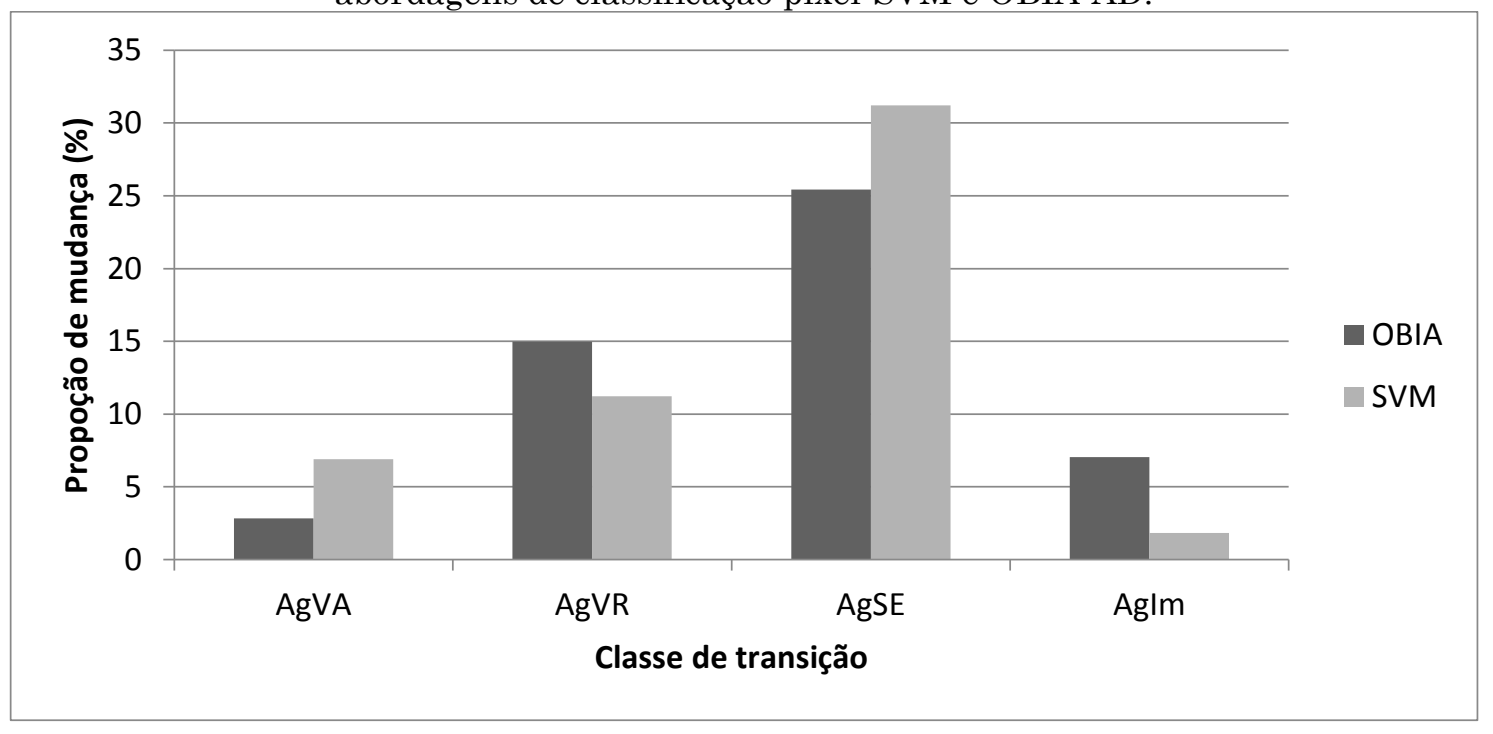

Fonte: Elaborado pelos autores.

A Figura 5 corrobora a percepção possibilitada pelos mapas da Figura 4, de que a detecção de áreas impermeáveis foi consideravelmente maior na classificação OBIA-AD. Por levar a uma atribuição equivocada no tipo de uso 
e não apenas decorrente de uma confusão quanto ao grau de cobertura do solo pela vegetação (arbórea ou arbustiva), como ocorre na classificação pixelSVM, pode-se considerar que a abordagem OBIA-AD produziu um resultado menos consistente.

\section{Conclusões}

A informação produzida pela classificação de imagens mostrou-se adequada para o monitoramento de recursos hídricos, resultando em mapeamentos de alta acurácia temática. Os tipos de cobertura da terra associados a corpos d'água, vegetação arbórea, vegetação rasteira, solo exposto e áreas impermeáveis foram discriminados tanto pela abordagem de classificação pixel-SVM, como a OBIA-AD para as imagens tomadas em 2013 e 2014.

$\mathrm{Na}$ análise da qualidade das classificações resultantes foram consideradas as concordâncias e discrepâncias expressas na matriz de confusão de cada classificação produzida e verificou-se que, com exceção da classe área impermeável (Im), a discriminação dos demais tipos de cobertura apresentou elevada concordância com os dados de referência. A comparação do desempenho entre os métodos de classificação empregados indicou que a abordagem baseada em pixel resultou em indicadores de acurácia superiores aos do OBIA, em 2013 e 2014 e, portanto, mostrou-se mais adequada para o mapeamento temático dos tipos de cobertura da terra no entorno do reservatório Jaguari-Jacareí, mesmo que as classificações tenham sido estatisticamente similares, exceto pixel-SVM de 2014.

A análise das mudanças ocorridas na paisagem de entorno do reservatório Jaguari-Jacareí durante a crise hídrica, realizada a partir da interseç̧ão dos mapas temáticos de 2013 e 2017 produzidos pelos classificadores pixel-SVM e OBIA-AD, individualmente, indicou os tipos de cobertura que ocuparam as margens do reservatório com a redução da superfície do corpo d'água durante a crise hídrica. Enquanto o classificador 
pixel-SVM mapeou como solo exposto e vegetação rasteira ou arbórea áreas antes (em 2013) ocupadas por água, a abordagem OBIA-AD resultou em uma área quase quatro vezes maior atribuída a área impermeável. Essa constatação é desfavorável à OBIA-AD, haja vista que os únicos objetos impermeáveis presentes na área são a infraestrutura da barragem, píeres e o afloramento rochoso do canal que interliga os rios Jaguari e Jacareí. Em relação à detecção do corpo d'água, ambas as abordagens resultaram em áreas similares em 2013 (35,37 km² por OBIA-AD contra $36,24 \mathrm{~km}^{2}$ por pixel-SVM) e $2014\left(17,51 \mathrm{~km}^{2}\right.$ por OBIA-AD e $17,46 \mathrm{~km}^{2}$ por pixel-SVM), indicando que teve sua superfície reduzida em quase $50 \%$ com a crise hídrica.

\section{Agradecimentos}

Os autores agradecem à Superintendência de Pesquisa, Desenvolvimento Tecnológico e Inovação da Sabesp (Companhia de Saneamento Básico do Estado de São Paulo) e ao Ministério do Meio Ambiente por disponibilizar as imagens RapidEye usadas nesta investigação. O primeiro autor agradece à CAPES (Coordenação de Aperfeiçoamento de Pessoal de Nível Superior) pela concessão de bolsa de mestrado junto ao Programa de Pós-graduação de Ciências Cartográficas da Universidade Estadual Paulista Júlio de Mesquita Filho.

\section{Referências}

ANA, Agência Nacional de Águas. Site $<$ http://audienciapublica.ana.gov.br/arquivos/Aud_001_2014_DadosdeReferen ciaAcercadaOutorgadoSistemaCantareira.pdf>, acessado em 15 julho de 2016 . ALSDORF, D.; RODRÍGUEZ, E.; LETTENMAIER, D. Measuring surface water from space. Reviews of Geophysics, vol. 45, i. 2, 2007. pp. 1-24 
CÂMARA MUNICIPAL DA ESTÂNCIA TURÍSTICA DE JOANÓPOLIS. Site: <http://www.camarajoanopolis.sp.gov.br/planodiretor>, Acessado em agosto de 2016.

CONGALTON, R; GREEN, K. Assessing the accuracy of remotely sensed data: Principles and practices. Boca Raton: CRC Press, 1999, pp. 43-53.

DURO, D.; FRANKLIN, S.; DUBÉ, M. A comparison of pixel-based and object-based image analysis with selected machine learning algorithms for the classification of agricultural landscapes using SPOT-5 HRG imagery. Remote Sensing of Environment, vol. 118, 2012. pp. 259-272

HAN, X.; CHEN, X.; FENG, L. Four decades of winter wetlands changes in Poyang Lake based on Landsat observations between 1973 and 2013. Remote Sensing of Environment, vol. 156, 2015. pp. 426-437

JENSEN, J. Sensoriamento remoto do ambiente: uma perspectiva em recursos terrestres. Tradução de José Carlos Neves Epiphanio (coordenador).. [et al.]. São José dos Campos: Parêntese, 2011.

KALKAN, K.; BAYRAM, B.; MAKTAV, D.; SUNAR, F. Comparison of support vector machine and object base classification methods for coastline detection. Proceeding of the International Archives of the Photogrammetry, Remote Sensing and Spatial Information Sciences, Antalya, 2013. pp. $125-127$

KHATAMI, R.; MOUNTRAKIS, G.; STEHMAN, S. A meta-analysis of remote sensing research on supervised pixel-based land-cover image classification processes: General guidelines for practitioners and future research. Remote Sensing of Environment, vol. 177, 2016. pp. 89-100

LALIBERTE, A.; FREDRICKSON, E.; RANGO, A. Combining decision trees with hierarchical object-oriented image analysis for mapping arid rangelands. Photogrammetric Engineering \& Remote Sensing, v. 73, n. 2, 2007. pp. 197-207

MARENGO, J.; NOBRE, C.; SELUCHI, M.; CUARTAS, A.; ALVES, L.; MEDIONDO, E.; OBREGÓN, G.; SAMPAIO, G. A seca e a crise hídrica de 2014-2015 em São Paulo. Revista USP, n. 106, 2015. pp. 31-44 
MOUNTRAKIS, G.; IM, J.; OGOLE, C. Support vector machines in remote sensing: A review. ISPRS Journal of Photogrammetry and Remote Sensing. vol. 66, 2011. pp. 247-259

MUALA, E.; MOHAMED, Y.; DUAN, Z.; VAN DER ZAAG, P. Estimation of reservoir discharges from Lake Nasser and Roseires reservoir in the Nile basin using satellite altimetry and imagery data. Remote Sensing, vol. 6, 2014. pp. 7522 7545

MYINT, S; GOBER, P.; BRAZEL, A.; GROSSMAN-CLARKE, S., WENG, Q. Perpixel vs. object-based classification of urban land cover extraction using high spatial resolution imagery. Remote Sensing of Environment, vol. 115, 2011. pp. 1145-1161

NOBRE, C.; MARENGO, J.; SELUCHI, M.; CUARTAS, A.; ALVES, L. Some characteristics and impacts of the drought and water crisis in southeastern Brazil during 2014 and 2015. Journal of Water Resource and Protection, vol. 8, 2016. pp. 252-262

PLANET. Site <https://www.planet.com/products/satellite-imagery/files/160625RapidEye\%20Image-Product-Specifications.pdf>, acessado em 15 de janeiro de 2017.

PREFEITURA DO MUNICÍPIO DE BRAGANÇA PAULISTA. Site <http://www.braganca.sp.gov.br/newsite/content.php?mid=119\&unc=1470237 855>, acessado em 15 de agosto de 2016.

$\begin{array}{llll}\text { PREFEITURA } & \text { MUNICIPAL }\end{array}$ <http://www.piracaia.sp.gov.br/novo_site/plano_diretor/documentos/2007/2010 0407122125.pdf $>$, acessado em 15 de agosto de 2016 .

$\begin{array}{lllll}\text { PREFEITURA DO } & \text { MUNICÍPIO }\end{array}$ $<$ http://www.vargem.sp.gov.br/novo_site/index.php?nivel=0\&exibir=plano_dir etor\&ID=38>, acessado em 15 de agosto de 2016 .

SABESP.

Site

<http://www2.Sabesp.com.br/mananciais/DivulgacaoSiteSabesp.aspx>, acessado em 27 de julho de 2016.

SCHWEIE, J.; TUFTE, L. O potencial de procedimentos baseados em regiões para a avaliação integrada de dados de SIG e sensoriamento remoto. In: BLASCHKE, 
T.; KUX, H. (Orgs.). Sensoriamento remoto e SIG avançados. São Paulo: Oficina de Textos, 2007. pp. 56-65.

VAPNIK, V. Statistical Learning Theory. New York: John Wiley \& Sons, Inc., 1998.

WHITESIDE, T.; BOGGS, G.; MAIER, S. Comparing object-based and pixel-based classifications for savannas. International Journal of Applied Earth Observation and Geoinformation, vol. 13, 2011. pp. 884-893

ZHU, W.; JIA, S.; LV, A. Monitoring the fluctuation of lake Qinghai using multisource remote sensing data. Remote Sensing, vol. 6, 2014. pp. 10457-10482 